\title{
Association of subclinical inflammation with deterioration of glycaemia before the diagnosis of type 2 diabetes: the KORA S4/F4 study
}

\author{
Birgit Klüppelholz $^{1}$ - Barbara Thorand ${ }^{2,3}$ - Wolfgang Koenig ${ }^{4}$. \\ Tonia de las Heras Gala ${ }^{2,3}$. Christa Meisinger ${ }^{2}$. Cornelia Huth ${ }^{2,3}$ • \\ Guido Giani $^{1,5}$ - Paul W. Franks ${ }^{6,7,8}$ - Michael Roden ${ }^{9,10,11}$. \\ Wolfgang Rathmann ${ }^{1}$ - Annette Peters ${ }^{2,3}$. Christian Herder ${ }^{9,10}$
}

Received: 1 June 2015 / Accepted: 3 June 2015 /Published online: 9 July 2015

(C) Springer-Verlag Berlin Heidelberg 2015

\begin{abstract}
Aims/hypothesis The role of biomarkers of subclinical inflammation in the early deterioration of glycaemia before type 2 diabetes is largely unknown. We hypothesised that increased levels of circulating proinflammatory biomarkers and decreased circulating adiponectin would be associated with 7 year increases of $\mathrm{HbA}_{1 \mathrm{c}}$ in non-diabetic individuals.

Methods This study was based on individuals who participated in the prospective Cooperative Health Research in the Region of Augsburg (KORA) S4 survey (1999-2001) and the 7 year follow-up KORA F4 (2006-2008) survey. Individuals with type 2 diabetes at baseline or with a diagnosis of diabetes in the period between both surveys were excluded, which left a sample of 850 men and women. Multivariable linear regression analyses were performed to
\end{abstract}

Electronic supplementary material The online version of this article (doi:10.1007/s00125-015-3679-4) contains peer-reviewed but unedited supplementary material, which is available to authorised users.

Christian Herder

christian.herder@ddz.uni-duesseldorf.de

1 Institute for Biometrics and Epidemiology, German Diabetes Center, Leibniz Center for Diabetes Research at Heinrich Heine University Düsseldorf, Düsseldorf, Germany

2 Institute of Epidemiology II, Helmholtz Zentrum München German Research Center for Environmental Health, Neuherberg, Germany

3 German Center for Diabetes Research, Neuherberg, Germany

4 Department of Internal Medicine II - Cardiology, University of Ulm Medical Center, Ulm, Germany

5 Institute for Statistics in Medicine, University Hospital Düsseldorf, Düsseldorf, Germany assess associations among baseline values of leucocyte count and levels of acute-phase proteins (high-sensitivity C-reactive protein [hsCRP], serum amyloid A [SAA] and fibrinogen), IL-6 and adiponectin with changes in $\mathrm{HbA}_{1 \mathrm{c}}$ between baseline and follow-up.

Results A high leucocyte count and high hsCRP, SAA and IL-6 levels were positively associated with changes in $\mathrm{HbA}_{1 \mathrm{c}}$ after adjusting for age, sex, lifestyle factors and baseline $\mathrm{HbA}_{1 \mathrm{c}}$. In contrast, the adiponectin level was inversely associated with changes in $\mathrm{HbA}_{1 \mathrm{c}}$ ( $p$ value between $<0.0001$ and 0.020 ). The associations of leucocyte count and levels of hsCRP and SAA with $\mathrm{HbA}_{1 \mathrm{c}}$ changes remained significant after additional adjustment for waist circumference and circulating lipids at baseline and for the 7 year change in waist circumference ( $p$ value between 0.004 and 0.045 ).

Genetic \& Molecular Epidemiology Unit, Lund University Diabetes Center, Department of Clinical Sciences, Skåne University Hospital Malmö, Lund University, Malmö, Sweden

7 Department of Public Health \& Clinical Medicine, Umeå University, Umeå, Sweden

8 Department of Nutrition, Harvard School of Public Health, Boston, MA, USA

9 Institute of Clinical Diabetology, German Diabetes Center, Leibniz Center for Diabetes Research at Heinrich Heine University Düsseldorf, Auf'm Hennekamp 65, 40225 Düsseldorf, Germany

10 German Center for Diabetes Research, Düsseldorf, Germany

11 Department of Endocrinology and Diabetology, University Hospital Düsseldorf, Düsseldorf, Germany 
Conclusions/interpretation An elevated leucocyte count and elevated hsCRP and SAA were associated with early deterioration of glycaemia before the diagnosis of type 2 diabetes. These associations were largely independent of baseline abdominal adiposity and increases in waist circumference.

Keywords Adiponectin - Cohort study · Cytokines . Glycaemia $\cdot \mathrm{HbA}_{1 \mathrm{c}} \cdot$ Inflammation $\cdot$ Type 2 diabetes

\begin{tabular}{|c|c|}
\hline \multicolumn{2}{|c|}{ Abbreviations } \\
\hline CARDIA & $\begin{array}{l}\text { Coronary Artery Risk Development on Young } \\
\text { Adults }\end{array}$ \\
\hline DIRECT & Diabetes Research on Patient Stratification \\
\hline hsCRP & High-sensitivity C-reactive protein \\
\hline IFG & Impaired fasting glucose \\
\hline IGT & Impaired glucose tolerance \\
\hline KORA & $\begin{array}{l}\text { Cooperative Health Research in the Region of } \\
\text { Augsburg }\end{array}$ \\
\hline NFG & Normal fasting glucose \\
\hline NGT & Normal glucose tolerance \\
\hline SAA & Serum amyloid A \\
\hline
\end{tabular}

\section{Introduction}

Subclinical inflammation represents an important mechanism in the development of type 2 diabetes [1]. Data from epidemiological studies have demonstrated that increased levels of proinflammatory immune mediators and decreased levels of the anti-inflammatory adipokine adiponectin precede the onset of diabetes by many years [1-3]. Several approaches to attenuate subclinical inflammation in type 2 diabetes have improved glycaemic control [4-8], underscoring the clinical relevance of the inflammatory processes in the regulation of glucose metabolism.

However, most studies that have investigated subclinical inflammation in the context of type 2 diabetes have focused on the incidence of diabetes as outcome, whereas little is known about the role of proinflammatory processes such as impaired fasting glucose (IFG) or impaired glucose tolerance (IGT) in the early stages of dysglycaemia; together, these processes are termed 'prediabetes' [9].

A better understanding of subclinical inflammation in prediabetes is important because (1) prediabetes is highly prevalent in most adult populations worldwide [10-12]; and (2) individuals with prediabetes have a moderately increased risk of macrovascular and microvascular complications [9] which were both previously considered diabetic complications in the later stages of the disease.

A range of cross-sectional studies have observed elevated levels of acute-phase proteins and proinflammatory cytokines and decreased levels of adiponectin in individuals with prediabetes; the levels of these biomarkers are usually intermediate between those observed in normoglycaemic individuals and in patients with manifest type 2 diabetes [13, 14]. Only a few prospective studies have tested the hypothesis that circulating levels of immune mediators can predict the deterioration of glycaemic control before the manifestation of type 2 diabetes [15-17]. In two studies, deterioration of glycaemia was assessed as the incidence of IFG or IGT $[15,16]$; another study used increases in HOMA-IR as the outcome [17].

The recent incorporation of $\mathrm{HbA}_{1 \mathrm{c}}$ measurements into guidelines for the diagnosis of type 2 diabetes or for the identification of individuals with prediabetes raises the question of whether biomarkers of subclinical inflammation are associated with increased $\mathrm{HbA}_{1 \mathrm{c}}$ in the prediabetic phase, in addition to predicting the onset of type 2 diabetes. Therefore, the primary aim of our study was to assess the associations of different immunological variables (leucocyte count and levels of three acute-phase proteins, IL-6 and adiponectin) with changes in $\mathrm{HbA}_{1 \mathrm{c}}$ over a follow-up of 7 years. Since obesity is an important determinant of both subclinical inflammation and glycaemic control [18], the secondary aim of our study was to investigate the extent to which baseline levels of abdominal adiposity and 7 year changes in waist circumference may explain these associations.

\section{Methods}

Study population Data are based on the population-based Cooperative Health Research in the Region of Augsburg (KORA) S4 (1999-2001) and the 7 year follow-up KORA F4 surveys (2006-2008), which were conducted in Augsburg and two adjacent counties of Southern Germany. The design of both surveys has been described $[19,20]$.

KORA S4 recruited study participants aged 25-74 years, but OGTTs were only performed for those aged $55-74$ years. The current study is based on 850 individuals aged 55-74 years (Fig. 1). Of the initial study sample of 1,653 individuals in this age range who participated in KORA S4, follow-up data from KORA F4 were unavailable for 492 individuals (because of death, declined participation or loss to follow-up for other reasons; electronic supplementary material [ESM] Table 1). Of the remaining 1,161 individuals, 133 were excluded because of unclear glucose tolerance status in either KORA S4 or KORA F4. A further 174 individuals were excluded because of known or newly diagnosed diabetes in KORA S4 $(n=141)$ or a diagnosis of diabetes in the interval between KORA S4 and KORA $\mathrm{F} 4(n=33)$. Of the remaining 854 individuals who were initially non-diabetic and did not report a diagnosis of diabetes between baseline and follow-up, four were excluded because of missing values for $\mathrm{HbA}_{1 \mathrm{c}}$ in either KORA S4 or KORA F4, which left a final study sample of 850 . 


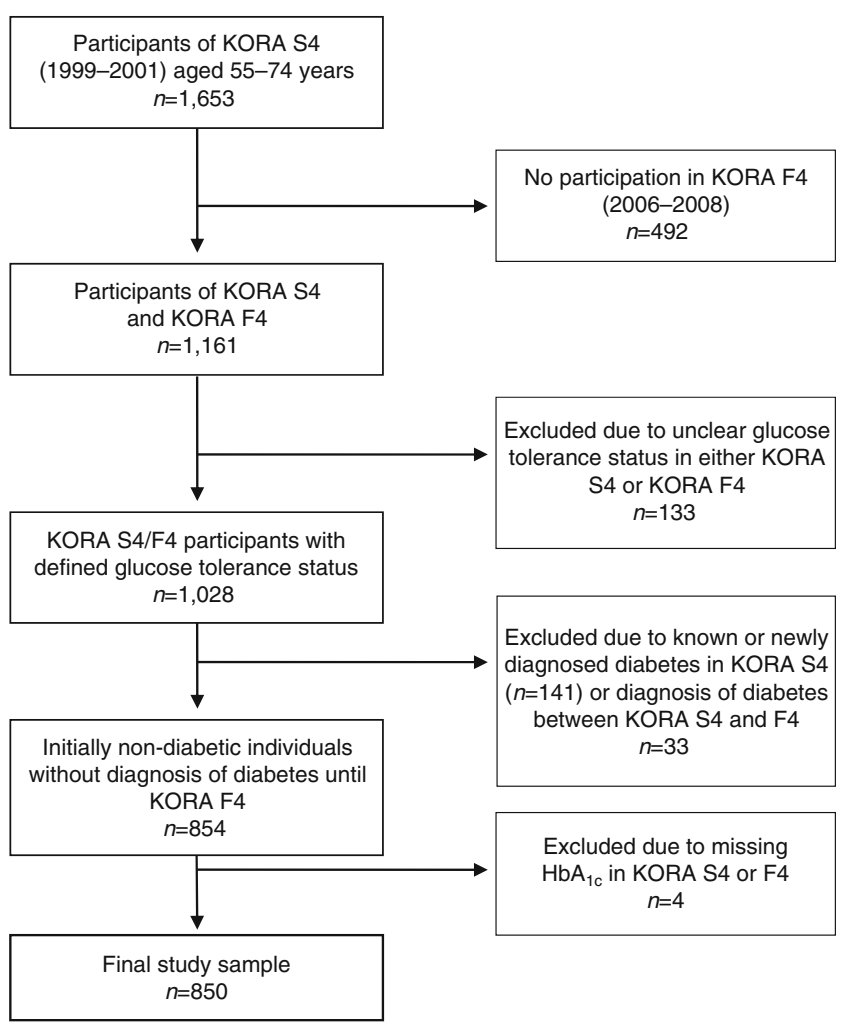

Fig. 1 Flowchart showing the final study population after exclusions

All participants gave written informed consent, and the study was approved by the Ethics Committee of the Bavarian Medical Association.

\section{Assessment of anthropometric, metabolic and lifestyle variables Height, weight and waist circumference were mea- sured according to standardised protocols $[19,20]$. Trained med- ical interviewers collected information on the medical history and lifestyle factors of participants, including physical activity, smoking behaviour and alcohol consumption in both surveys $[19,20]$. Study participants were designated 'physically active' if they engaged in $\geq 1 \mathrm{~h}$ of leisure time physical activity per week in both summer and winter. Alcohol consumption was classified into three groups: none, moderate ( $>0$ to $<40 \mathrm{~g} /$ day for men, $>0$ to $<20 \mathrm{~g} /$ day for women) or high ( $\geq 40 \mathrm{~g} /$ day for men, $\geq 20 \mathrm{~g} /$ day for women), as in previous KORA analyses [20]. \\ Glucose tolerance status was assessed in KORA S4 with 75 g OGTTs using the 1999 WHO diagnostic criteria for fasting and $2 \mathrm{~h}$ glucose levels [21]. OGTTs were performed for all individuals without known type 2 diabetes after an overnight fasting period of $\geq 8 \mathrm{~h}$ [19]. Glucose and lipid levels (LDL-cholesterol, HDL-cholesterol and triacylglycerols) were measured as described $[13,19]$.}

Measurement of $\mathbf{H b A}_{\mathbf{1 c}}$ In KORA S4, $\mathrm{HbA}_{1 \mathrm{c}}$ was determined using a turbidimetric immunological method (Tinaquant; Roche Diagnostics, Mannheim, Germany) with a
Hitachi 717 analyser. In KORA F4, $\mathrm{HbA}_{1 \mathrm{c}}$ was quantified with a reverse-phase cation-exchange HPLC method using a Menarini-Arkray Analyzer HA-8140 (Menarini Diagnostics, Florence, Italy).

Calibration measurements for calculating interassay conversion factors were not performed in the KORA studies. However, one study compared the Tina-quant method and the HPLC assay on the Menarini-Arkray Analyzer HA-8160 and obtained the following regression equation for the measured $\mathrm{HbA}_{1 \mathrm{c}}$ values (in \%): $y=0.99 x-0.06$, with $x=$ Tina-quant and $y=\mathrm{HA}-8140$ [22]. A second study compared the HPLC assays on the HA- 8140 and HA- 8160 analysers and observed $y=1.1038 x-$ 0.718 , with $x=\mathrm{HA}-8140$ and $y=\mathrm{HA}-8160$ [23]. The use of both equations allowed transformation of the $\mathrm{HbA}_{1 \mathrm{c}}$ values from KORA S4 (Tina-quant) to those of the HA- 8160 based method used in KORA F4. All main analyses were performed using the transformed baseline $\mathrm{HbA}_{1 \mathrm{c}}$ values. Sensitivity analyses using untransformed baseline $\mathrm{HbA}_{1 \mathrm{c}}$ levels were also carried out.

Measurement of immunological variables Immunological variables were measured in KORA S4 according to the following methods. The leucocyte count was determined using a Coulter STKS Hematology Analyzer (Block Scientific, New York, NY, USA). Plasma concentrations of high-sensitivity C-reactive protein (hsCRP) were assessed using a highsensitivity latex-enhanced nephelometric assay on a BN II System analyser (Dade Behring, Marburg, Germany) [13]. Plasma serum amyloid A (SAA) and fibrinogen were determined by immunonephelometry [13]. Serum levels of IL-6 were measured using the PeliKine Compact human IL-6 ELISA Kit (CLB, Amsterdam, the Netherlands) [13]. Serum levels of adiponectin were assessed using the human adiponectin RIA from Linco Research (St. Charles, MO, USA) [24]. The intra-assay coefficients of variation for hsCRP, SAA, fibrinogen, IL-6 and adiponectin levels were $2.1 \%, 6.7 \%, 3.6 \%,<10 \%$ and $5.5 \%$, respectively; interassay coefficients of variation were $5.9 \%, 5.6 \%, 4.6 \%,<10 \%$ and $9.2 \%$, respectively. For 57 serum samples, IL-6 levels were below the limit of detection of $0.24 \mathrm{pg} / \mathrm{ml}$, and were set at $50 \%$ of the detection limit (i.e. $0.12 \mathrm{pg} / \mathrm{ml}$ ) for all analyses.

Statistical analysis Baseline characteristics of the study cohort are presented as median (25th, 75th percentiles) for continuous variables and percentages for categorical variables. Correlations between baseline levels of immune mediators, anthropometric and metabolic variables were estimated using Pearson $r$ coefficients. Variables without normal distribution were $\log _{e}$-transformed prior to analysis.

Associations between baseline immune mediators and 7 year changes in $\mathrm{HbA}_{1 \mathrm{c}}$ were assessed using multivariable linear regression models. The change in $\mathrm{HbA}_{1 \mathrm{c}}\left(\mathrm{HbA}_{1 \mathrm{c}}[\mathrm{F} 4]\right.$ minus $\mathrm{HbA}_{1 \mathrm{c}}[\mathrm{S} 4]$, both in \%) was used as the dependent variable and immune mediator levels as independent variables. In 
order to approximate normal distributions, immune mediator levels (except for leucocyte count), and $\mathrm{HbA}_{1 c}[\mathrm{~S} 4]$, HDLcholesterol and triacylglycerol levels were $\log _{e}$-transformed. All independent variables are based on KORA S4 (baseline), unless indicated otherwise. Model 1 was adjusted for age, sex and $\mathrm{HbA}_{1 \mathrm{c}}[\mathrm{S} 4]$. Model 2 also included smoking (ever/never), physical activity (active/inactive) and alcohol intake (none/moderate/high). Model 3 extended Model 2 by also adjusting for waist circumference and LDL-cholesterol, HDL-cholesterol and triacylglycerol levels. Model 4 additionally adjusted for changes in waist circumference (waist circumference[F4] minus waist circumference[S4]). Model 5 extended Model 4 by including a family history of diabetes (positive family history defined as diagnosed diabetes in mother and/or father). Analogous models adjusting for baseline levels and changes in BMI instead of waist circumference were calculated as sensitivity analyses. To assess the variance in $\mathrm{HbA}_{1 \mathrm{c}}$ changes that was explained by baseline levels of inflammation-related biomarkers, squared partial correlation coefficients $\left(r^{2}\right)$ were determined for all regression models.

A $p$ value of $<0.05$ was considered statistically significant without adjustment for multiple testing because of the varying degrees of correlation between the immune mediator levels as exposure variables. All analyses were performed with SAS statistical software (version 9.2, SAS Institute, Cary, NC, USA).

\section{Results}

Study population at baseline Figure 1 depicts the selection of the study population, starting with all participants of KORA S4 aged 55-74 years. As described in ESM Table 1, nonparticipants in KORA F4 were older, more obese and had less favourable glucose tolerance and lifestyle factors as well as higher levels of triacylglycerols and most inflammationrelated biomarkers compared with individuals who participated in KORA F4.

The baseline characteristics of the 850 non-diabetic study participants who comprise the study sample are shown in Table 1. Individuals were aged 55-74 years, most were overweight or obese, and $24.8 \%$ had prediabetes as defined by IFG, IGT or combined IFG/IGT.

There were positive correlations among leucocyte count and levels of acute-phase proteins (hsCRP, SAA, fibrinogen) and IL- 6 ( $r$ between 0.20 and 0.61 , all $p<0.001$ ). Adiponectin levels showed weaker, inverse correlations with leucocyte count $(r=-0.15)$ and hsCRP $(r=-0.08)$ only (Table 2$)$.
Table 1 Description of the baseline characteristics of the study population (KORA S4, 19992001)

\begin{tabular}{|c|c|}
\hline Variable & Value \\
\hline Age (years) & $63(58,67)$ \\
\hline Sex, male/female (\%) & $49.5 / 50.5$ \\
\hline Waist circumference $(\mathrm{cm})$ & $94.3(86.5,101.0)$ \\
\hline BMI $\left(\mathrm{kg} / \mathrm{m}^{2}\right)$ & $27.6(25.4,30.1)$ \\
\hline $\mathrm{HbA}_{1 \mathrm{c}}(\%)$ & $5.34(5.12,5.55)$ \\
\hline $\mathrm{HbA}_{1 \mathrm{c}}(\mathrm{mmol} / \mathrm{mol})$ & $35(32,37)$ \\
\hline Glucose tolerance status, NGT/IFG/IGT/IFG+IGT (\%) & $75.2 / 7.9 / 12.8 / 4.1$ \\
\hline LDL-cholesterol (mmol/l) & $3.99(3.32,4.71)$ \\
\hline HDL-cholesterol (mmol/1) & $1.48(1.23,1.79)$ \\
\hline Triacylglycerols (mmol/l) & $1.24(0.99,1.72)$ \\
\hline Smoking, current or former/never (\%) & $47.4 / 52.6$ \\
\hline Alcohol consumption, none/moderate/high ${ }^{\mathrm{a}}(\%)$ & $23.8 / 55.4 / 20.8$ \\
\hline Physical activity, active/inactive ${ }^{\mathrm{b}}(\%)$ & $47.8 / 52.2$ \\
\hline \multicolumn{2}{|l|}{ Biomarkers of subclinical inflammation } \\
\hline Leucocytes $\left(\times 10^{-3} / \mu \mathrm{l}\right)$ & $5.7(4.9,6.6)$ \\
\hline $\mathrm{hsCRP}(\mathrm{nmol} / \mathrm{l})$ & $14(8,29)$ \\
\hline $\mathrm{SAA}(\mathrm{mg} / \mathrm{l})$ & $3.5(2.3,5.8)$ \\
\hline Fibrinogen $(\mathrm{g} / \mathrm{l})$ & $2.8(2.4,3.2)$ \\
\hline IL-6 (pg/ml) & $1.7(0.9,2.8)$ \\
\hline Total adiponectin $(\mu \mathrm{g} / \mathrm{ml})$ & $9.1(6.4,12.3)$ \\
\hline
\end{tabular}

Data are medians (25th, 75 th percentiles) or percentages $(n=850)$

${ }^{a}$ Moderate is defined as $>0$ to $<40 \mathrm{~g} /$ day for men, $>0$ to $<20 \mathrm{~g} /$ day for women; high is defined as $\geq 40 \mathrm{~g} /$ day for men and $\geq 20 \mathrm{~g} /$ day for women

${ }^{\mathrm{b}}$ Active defined as $\geq 1 \mathrm{~h}$ leisure time physical activity/week in summer and in winter 
Table 2 Pearson $r$ values for baseline levels of immune mediators with one another, $\mathrm{HbA}_{1 \mathrm{c}}$, lipids and adiposity

\begin{tabular}{lllllll}
\hline Variable & Leucocytes & hSCRP & SAA & Fibrinogen & IL-6 & Adiponectin \\
\hline WC & $0.11^{* *}$ & $0.22^{* * *}$ & 0.02 & $0.10^{* *}$ & $0.17^{* * *}$ & $-0.31^{* * *}$ \\
BMI & $0.09^{* *}$ & $0.28^{* * *}$ & $0.13^{* * *}$ & $0.18^{* * *}$ & $0.16^{* * *}$ & $-0.11^{* *}$ \\
$\mathrm{HbA}_{1 \mathrm{c}}$ & $0.11^{* *}$ & $0.08^{*}$ & 0.05 & $0.16^{* * *}$ & -0.01 & -0.05 \\
LDL-C & 0.03 & 0.05 & 0.02 & $0.13^{* * *}$ & 0.03 & -0.05 \\
HDL-C & $-0.17^{* * *}$ & $-0.12^{* * *}$ & $0.08^{*}$ & $-0.10^{* *}$ & $-0.09^{* *}$ & $0.41^{* * *}$ \\
Triacylglycerols & $0.18^{* * *}$ & $0.16^{* * *}$ & 0.03 & 0.01 & $0.11^{* *}$ & $-0.27^{* * *}$ \\
Leucocytes & 1 & $0.33^{* * *}$ & $0.20^{* * *}$ & $0.20^{* * *}$ & $0.21^{* * *}$ & $-0.15^{* * *}$ \\
hsCRP & - & 1 & $0.61^{* * *}$ & $0.46^{* * *}$ & $0.35^{* * *}$ & $-0.08^{*}$ \\
SAA & - & - & 1 & $0.36^{* * *}$ & $0.23^{* * *}$ & 0.06 \\
Fibrinogen & - & - & - & 1 & $0.22^{* * *}$ & 0.01 \\
IL-6 & - & - & - & - & 1 & 0.02 \\
Adiponectin & - & - & - & - & - & 1 \\
\hline
\end{tabular}

Data are Pearson's $r$ coefficients

All variables except waist circumference, leucocyte count, BMI and LDL-cholesterol level were $\log _{e^{-}}$ transformed

${ }^{*} p<0.05,{ }^{* *} p<0.01, * * * p<0.001$

HDL-C, HDL-cholesterol; LDL-C, LDL-cholesterol; WC, waist circumference
When correlations with waist circumference, $\mathrm{BMI}, \mathrm{HbA}_{1 \mathrm{c}}$ and lipids (triacylglycerols, HDL and LDL-cholesterol) were assessed, the strongest relationships were found between adiponectin and both HDL-cholesterol $(r=0.41)$ and waist circumference $(r=-0.31)$ and between hsCRP and both waist circumference $(r=0.22)$ and BMI $(r=0.28)$; all other $r$ values were between -0.2 and 0.2 (Table 2 ).

\section{Changes in $\mathrm{HbA}_{1 \mathrm{c}}$, glucose levels and waist circumference} between baseline and 7 year follow-up $\mathrm{HbA}_{1 \mathrm{c}}$ (median; 25 th, 75th percentiles) at baseline was $5.34 \%(5.12 \%$, $5.55 \%) ; 35(32,37) \mathrm{mmol} / \mathrm{mol}$ and increased by $0.28 \%$ $(0.02 \%, 0.58 \%) ; 3.1(0.2,6.3) \mathrm{mmol} / \mathrm{mol}$ until the 7 year follow-up $(p<0.001)$; thus, the mean increase in $\mathrm{HbA}_{1 \mathrm{c}}$ was $0.04 \%(0.4 \mathrm{mmol} / \mathrm{mol})$ per year. Fasting glucose levels did not change from $5.44(5.11,5.77) \mathrm{mmol} / \mathrm{l}$ at baseline (difference during the follow-up $0[-0.28,0.28] \mathrm{mmol} / \mathrm{l}, p=0.973), 2 \mathrm{~h}$ glucose levels from the OGTT rose from $5.99(5.05,7.10)$ $\mathrm{mmol} / \mathrm{l}$ by $0.67(-0.44,2.00) \mathrm{mmol} / \mathrm{l}(p<0.001)$. Seven-year changes in $\mathrm{HbA}_{1 \mathrm{c}}$ were significantly correlated with changes in both fasting $(r=0.23, p<0.001)$ and $2 \mathrm{~h}$ glucose levels $(r=$ $0.09, p<0.01$ ).

During the same period, waist circumference increased from $94.3(86.5,101.1) \mathrm{cm}$ at baseline by $3.1(-0.5,6.4) \mathrm{cm}$ $(p<0.0001)$, equivalent to an increase of $0.44 \mathrm{~cm} /$ year. BMI increased from $27.6(25.4,30.1) \mathrm{kg} / \mathrm{m}^{2}$ at baseline by 0.27 $(-0.58,1.21) \mathrm{kg} / \mathrm{m}^{2}(p<0.001)$, with an average increase of $0.03 \mathrm{~kg} / \mathrm{m}^{2}$ per year.

Association of immunological variables at baseline with 7 year changes in $\mathbf{H b A}_{\mathbf{1 c}}$ As shown in Table 3, baseline leucocyte count and hsCRP, SAA and IL-6 levels were positively associated with changes in $\mathrm{HbA}_{1 \mathrm{c}}$ and adiponectin levels were inversely associated with changes in $\mathrm{HbA}_{1 \mathrm{c}}$ in Model 1 (adjusted for age, sex and baseline $\mathrm{HbA}_{1 \mathrm{c}}$ ). These associations were virtually unaltered after additional adjustment for lifestyle factors (smoking, physical activity, alcohol intake; Model 2). Effect sizes were reduced by $18-51 \%$ when baseline data for waist circumference and circulating lipids (LDL-cholesterol, HDL-cholesterol and triacylglycerols) were added in Model 3, but positive associations of baseline leucocyte counts and levels of hsCRP and SAA with increased $\mathrm{HbA}_{1 \mathrm{c}}$ during the follow-up remained significant ( $p$ value between 0.004 and 0.039 ). These associations were entirely independent of changes in waist circumference (Model 4).

Family history of type 2 diabetes is an important risk factor and may affect the aforementioned observations if it is associated with the genetic determinants of inflammationrelated biomarkers. Although the association between SAA level and changes in $\mathrm{HbA}_{1 \mathrm{c}}$ was not significant after additional adjustment for family history of type 2 diabetes in Model 5 ( $p=0.052)$, the addition of family history of type 2 diabetes to the model had almost no impact on effect estimates for all associations (Table 3).

In a first sensitivity analysis, all models were also calculated using untransformed baseline $\mathrm{HbA}_{1 \mathrm{c}}$ values, with almost identical results (ESM Table 2). In a second sensitivity analysis, waist circumference at baseline and follow-up was substituted with the corresponding BMI values; again, results were very similar to those of the main analysis (ESM Table 3).

Depending on the model, baseline leucocyte counts and levels of hsCRP and SAA explained $0.5-2.2 \%$ of the variance in 7 year changes in $\mathrm{HbA}_{1 \mathrm{c}}$ (ESM Table 4). In Model 4 , the main determinant of $\mathrm{HbA} 1 \mathrm{c}$ change was baseline 
Table 3 Association between immune mediators at baseline (KORA S4, 1999-2001) and changes in $\mathrm{HbA}_{1 \mathrm{c}}$ between baseline KORA S4 and 7 year follow up examination (KORA F4, 2006-2008)

\begin{tabular}{|c|c|c|c|c|c|c|c|c|c|c|}
\hline \multirow[t]{2}{*}{ Variable } & \multicolumn{2}{|c|}{ Model 1} & \multicolumn{2}{|c|}{ Model 2} & \multicolumn{2}{|l|}{ Model 3} & \multicolumn{2}{|c|}{ Model 4} & \multicolumn{2}{|c|}{ Model 5} \\
\hline & $\beta$ & $p$ & $\beta$ & $p$ & $\beta$ & $p$ & $\beta$ & $p$ & $\beta$ & $p$ \\
\hline Leucocytes $\left(\times 10^{-3} / \mu \mathrm{l}\right)$ & 0.175 & 0.001 & 0.185 & $<0.001$ & 0.145 & 0.005 & 0.149 & 0.004 & 0.147 & 0.004 \\
\hline hsCRP (nmol/l) & 0.045 & $<0.0001$ & 0.048 & $<0.0001$ & 0.033 & 0.004 & 0.031 & 0.007 & 0.031 & 0.007 \\
\hline $\mathrm{SAA}(\mathrm{mg} / \mathrm{l})$ & 0.036 & 0.016 & 0.039 & 0.011 & 0.032 & 0.039 & 0.031 & 0.045 & 0.030 & 0.052 \\
\hline Fibrinogen (g/l) & 0.097 & 0.077 & 0.087 & 0.125 & 0.053 & 0.358 & 0.050 & 0.383 & 0.056 & 0.325 \\
\hline IL-6 (pg/ml) & 0.022 & 0.034 & 0.025 & 0.020 & 0.014 & 0.188 & 0.011 & 0.305 & 0.012 & 0.272 \\
\hline Adiponectin $(\mu \mathrm{g} / \mathrm{ml})$ & -0.070 & 0.007 & -0.069 & 0.008 & -0.034 & 0.214 & -0.034 & 0.210 & -0.036 & 0.189 \\
\hline
\end{tabular}

$\beta$ coefficients and $p$ values are derived from linear regression analyses, with changes in $\mathrm{HbA}_{1 \mathrm{c}}\left(\mathrm{HbA}_{1 \mathrm{c}}[\mathrm{F} 4]\right.$ minus $\left.\mathrm{Hb} \mathrm{A}_{1 \mathrm{c}}[\mathrm{S} 4]_{\text {transformed }}\right)$ as dependent variable and $\log _{e}$-transformed immune mediator levels as independent variables (except for leucocyte count, which was not log-transformed).

Model 1: adjusted for age, sex and $\mathrm{HbA}_{1 \mathrm{c}}[\mathrm{S} 4]_{\text {transformed }}$

Model 2: Model 1+smoking, physical activity, alcohol intake

Model 3: Model 2+ waist circumference, LDL-cholesterol, HDL-cholesterol, triacylglycerols

Model 4: Model 3+changes in waist circumference (WC[F4] minus WC[S4])

Model 5: Model 4+family history of diabetes

$\mathrm{HbA}_{1 \mathrm{c}}\left(r^{2}=36.0-37.5 \%\right)$. Baseline waist circumference and change in waist circumference had comparable predictive values $\left(r^{2}=1.4-1.6 \%\right.$ for both variables $)$, age explained $0.8-1.0 \%$ of $\mathrm{HbA}_{1 \mathrm{c}}$ changes, and none of the other variables explained more than $0.6 \%$ of the variance on $\mathrm{HbA}_{1 \mathrm{c}}$ changes in Model 4 (data not shown).

\section{Discussion}

This study found that: (1) high leucocyte count and high levels of hsCRP, SAA and IL-6 were positively and adiponectin level was inversely associated with 7 year changes in $\mathrm{HbA}_{1 \mathrm{c}}$ in non-diabetic individuals; (2) associations of leucocyte count and hsCRP and SAA levels remained significant after adjustment for waist circumference and circulating lipids at baseline; and (3) these associations were entirely independent of concomitant changes in waist circumference between both survey periods.

\section{Associations between immunological variables and deteri-} oration of glycaemia This study extended the current literature in several respects. To the best of our knowledge, our study was the first to investigate long-term changes in $\mathrm{HbA}_{1 \mathrm{c}}$ in a non-diabetic study sample as the outcome. It also included six different biomarkers of subclinical inflammation which have been implicated in the development of type 2 diabetes. Finally, we were able to assess the impact of changes in adiposity during the study.

There are three previous studies to which our observations may be compared; they assessed changes in glycaemia or HOMA-IR as a surrogate measure of insulin resistance. The first study is the population-based Coronary Artery Risk Development on Young Adults (CARDIA) study, in which hsCRP levels in year 7 of the study were positively associated with HOMA-IR at years 15 and 20 after adjustment for age, sex, race, lifestyle factors, education and the respective baseline HOMA-IR; a similar association was found for hsCRP levels at year 15 and HOMA-IR at year 20 [17]. The mean age of the study population was 32 years at year 7 and 40 years at year 15. These data indicate that hsCRP levels in a young, non-diabetic cohort predict worsening of insulin resistance. However, given the causal link between insulin resistance and glycaemic control, the $\mathrm{HbA}_{1 \mathrm{c}}$ data from the KORA study are consistent with the CARDIA data for HOMA-IR. Both studies appear to differ with respect to fibrinogen, which showed significant associations with follow-up HOMA-IR values in CARDIA only [17], whereas the association with follow-up $\mathrm{HbA}_{1 \mathrm{c}}$ in KORA was not significant $(p=0.125$ after adjustment for age, sex, lifestyle factors and baseline $\mathrm{HbA}_{1 \mathrm{c}}$ ). This might reflect genuine differences in the strength of the associations between fibrinogen and the two outcome variables. Given the fact that both studies observed positive effect estimates, this difference could also be due to differences in sample size ( $n>2,000$ in CARDIA vs $n=850$ in KORA) and thus related to statistical power.

Two smaller studies provided data on associations between inflammation-related biomarkers and the incidence of IFG [16] or IGT [15]. The first of these reports from the Western New York Study compared the baseline hsCRP, IL-6 and adiponectin levels of individuals who progressed from normal fasting glucose (NFG) to IFG during a mean follow-up of 5.9 years with individuals who had NFG at both time points [16]. HsCRP levels were higher in men with IFG and 
adiponectin levels were lower in women with IFG, which is generally consistent with our data. No significant differences were observed for IL-6. Although tests for interaction by sex were not significant, combined analyses for men and women were not shown, and null findings of this study are somewhat difficult to interpret given the fairly small sample sizes (men: 39 cases, 117 controls; women: 52 cases, 156 controls). The second report from the Japanese Funagata Study included a comparison of adiponectin levels between 110 individuals who progressed from NGT to IGT during 5 years and 709 individuals with NGT at baseline and follow-up examinations [15]. No difference was found between the groups $(p=0.672)$. However, data were not adjusted for age or sex, which represent important confounders in this context, thus limiting the informative value of this observation. We are not aware of comparable studies that examined the association between leucocyte count or SAA level and early deterioration of glycaemia.

Our study is cross-sectional; therefore, we cannot infer causality. High leucocyte counts are associated with increased risk of type 2 diabetes [25, 26]; therefore, our data with respect to 7 year increases in $\mathrm{HbA}_{1 \mathrm{c}}$ in individuals with NGT or prediabetes are plausible. Differential white blood cell counts may help to improve our understanding of the underlying mechanisms responsible for this association. The Insulin Resistance Atherosclerosis Study indicated that lymphocytes in particular were associated with diabetes risk [26].

The causality of the relationship between hsCRP level and deterioration of glycaemic control is controversial. Mechanistic studies demonstrated that C-reactive protein induces insulin resistance in mice by inhibiting skeletal muscle glucose delivery [27]. In contrast, Mendelian randomisation studies showed that mild lifelong upregulation of CRP has no impact on $\mathrm{HbA}_{1 \mathrm{c}}$, HOMA-IR or incident type 2 diabetes [28]. The recent finding that infusion of pharmaceutical-grade, endotoxin-free human CRP had no significant clinical or proinflammatory effects, at least in healthy adults [29], also supports the view that elevated hsCRP levels may be considered a risk marker or indicator rather than a genuine risk factor.

SAA has been investigated less thoroughly, but we previously reported that high SAA levels are associated with increased risk of type 2 diabetes [30]. SAA induces the release of proinflammatory cytokines in different cell types and impairs insulin sensitivity in adipocytes [31, 32], so a causal relationship between SAA levels and worsening glycaemic control is sufficiently plausible to merit further study.

Hyperglycaemia and hyperlipidaemia are stimulators of inflammasomes [8, 33], and the finding that patients with type 2 diabetes have activated inflammasomes in myeloid cells [34] is intriguing. However, this study was based on a comparison of newly diagnosed diabetes patients and controls with mean $\mathrm{HbA}_{1 \mathrm{c}}$ levels of $8.5 \%$ and $5.0 \%$, respectively. Currently, it is not known to what extent mild hyperglycaemia at different stages of prediabetes can activate inflammasomes and thereby contribute to the immune activation seen in individuals at elevated diabetes risk.

Impact of adiposity on associations between immunological variables and deterioration of glycaemia Since there is a complex bidirectional relationship between adiposity and subclinical inflammation $[35,36]$ and since adiposity is the most dominant single risk factor of type 2 diabetes [37], the association between immunological variables and deterioration of glycaemic control cannot be investigated in a meaningful way without controlling for adiposity.

We observed that adjustment for baseline levels of waist circumference and circulating lipids in a combined model attenuated the associations between leucocyte counts and levels of hsCRP and SAA by $22 \%, 31 \%$ and $18 \%$, respectively, but did not abolish statistical significance. In contrast, the associations with IL-6 and adiponectin were explained to a larger extent ( $44 \%$ and $51 \%$, respectively) by these covariables. This means that, overall, $49-82 \%$ of the associations between immunological variables and changes in $\mathrm{HbA}_{1 \mathrm{c}}$ were independent of abdominal obesity and related lipid levels. Thus, in this study, the associations of hsCRP level as a predictor of $\mathrm{HbA}_{1 \mathrm{c}}$ changes were more robust than the associations of hsCRP level as a predictor of HOMA-IR for different follow-up periods in the CARDIA study; until now, the CARDIA study had included the most detailed analyses in this context. In the CARDIA study, adjustment for adiposity reduced effect estimates by $57-68 \%$ for waist circumference and by $45-53 \%$ for BMI [17]. One can speculate that adiposity is a stronger confounder of hsCRP levels at younger ages (e.g. in CARDIA), whereas subclinical inflammation in middleaged or older individuals (e.g. in KORA) is determined to a greater extent by adiposity-independent determinants. However, this cannot be directly inferred from the currently available data and needs further studies.

In addition to the correction for baseline adiposity, we demonstrated that further adjustment for 7 year changes of waist circumference or BMI had virtually no impact on effect estimates and did not confound the associations observed in our study. This issue has not been addressed in other studies so far and underscores the relevance of adiposity-independent determinants to the role of inflammation in the deterioration of glycaemia.

Determinants of $\mathrm{HbA}_{1 \mathrm{c}}$ changes in non-diabetic individuals Our data show that baseline levels of $\mathrm{HbA}_{1 \mathrm{c}}$ were by far the main determinants of subsequent $\mathrm{HbA}_{1 \mathrm{c}}$ changes in all models. The predictive values of leucocyte counts and levels of hsCRP and SAA were slightly lower than those of waist circumference and its changes, similar to the impact of age, and larger than the impact of all other covariables in our regression models. Comparable data are currently not available from other studies. 
However, it is interesting in this context that a recent crosssectional study of 8,088 Finnish men without type 2 diabetes found that hsCRP level explained $1.8 \%$ of $\mathrm{HbA}_{1 \mathrm{c}}$ variance, and was only exceeded by age (5.6\%) and fasting glucose $(4.2 \%)$ in a multiple linear regression model with an overall $R^{2}$ value of $17.1 \%$ [38]. These data emphasise that the variance of $\mathrm{HbA}_{1 \mathrm{c}}$ before the onset of type 2 diabetes is currently not well understood and that inflammation-related biomarkers are significant determinants of $\mathrm{HbA}_{1 \mathrm{c}}$ levels in non-diabetic individuals.

Strengths and limitations The main strengths of our study are the population-based design, the availability of six biomarkers reflecting different aspects of subclinical inflammation and the inclusion of changes in waist circumference or BMI during the study in our statistical analysis.

Our study also has several limitations that need to be mentioned here. First, two different $\mathrm{HbA}_{1 \mathrm{c}}$ assays were used, which limits the informative value of absolute changes in $\mathrm{HbA}_{1 \mathrm{c}}$ levels between baseline and follow-up studies. However, our analysis was designed to minimise this problem; our sensitivity analysis using untransformed $\mathrm{HbA}_{1 \mathrm{c}}$ baseline levels (ESM Table 2) strongly suggests that this methodological issue had no impact on our results. Second, waist circumference and BMI do not cover all aspects of adiposity and do not exclude the possibility of adiposity-related residual confounding. Unfortunately, estimates of body fat (as a percentage or in $\mathrm{kg}$ ) are only available for the KORA S4 study [35] and not for the follow-up KORA F4, which precluded the use of body fat content in the longitudinal analysis. Third, loss to follow-up (ESM Table 1) led to the study sample being slightly younger and healthier compared with the initial cohort of the KORA S4 study. Finally, the time difference between baseline and follow-up in the KORA study of 7 years did not allow us to study temporal relationships between changes in inflammationrelated biomarkers and changes in $\mathrm{HbA}_{1 \mathrm{c}}$ in detail, which might be useful for more precisely predicting the development of prediabetes or diabetes [39]. In order to better understand temporal trends in biomarkers and their impact on the development of prediabetes and type 2 diabetes, measurements at multiple time points are desirable. Such a study is currently ongoing within the Diabetes Research on Patient Stratification (DIRECT) Consortium under the banner of the Innovative Medicines Initiative, and will provide detailed information on the associations between biomarkers and the rate of glycaemic deterioration before the onset of type 2 diabetes, as well as after its diagnosis in several years [40].

Conclusion We demonstrated that a high leucocyte count and high levels of circulating hsCRP, SAA and IL-6 were positively and adiponectin level was inversely associated with 7 year changes in $\mathrm{HbA}_{1 \mathrm{c}}$ in an older and initially diabetesfree cohort. Associations of leucocyte count and levels of hsCRP and SAA remained significant after adjustment for baseline waist circumference, BMI and lipid levels; more importantly, they were not explained by concomitant 7 year changes in adiposity. Our data indicate that inflammationrelated processes, which are mainly independent of adiposity, also affect the deterioration of glycaemia before the diagnosis of type 2 diabetes. Considering these results in the context of studies on increased risk of macro- and microvascular comorbidities of prediabetes [9], our data furthermore suggest that interventions that attenuate subclinical inflammation in prediabetes may have clinical benefit and deserve more attention in the future.

Acknowledgements We thank U. Partke, G. Gornitzka, K. Röhrig and S. Müller-Scholze, German Diabetes Center, Düsseldorf, and G. Trischler, Ulm University, for excellent technical assistance. We appreciate the contribution of all study participants and are also grateful to the field staff in Augsburg who were involved in conducting the KORA S4 and F4 studies.

Funding This work was supported by the Ministry of Science and Research of the State of North Rhine-Westphalia and by the German Federal Ministry of Health. The diabetes part of the KORA S4 and F4 studies was funded by grants from the German Research Foundation (RA 459/3-1). This study was supported in part by a grant from the German Federal Ministry of Education and Research (BMBF) to the German Center for Diabetes Research. The KORA research platform and the KORA Augsburg studies are financed by the Helmholtz Zentrum München, German Research Center for Environmental Health, which is funded by the German Federal Ministry of Science and Research (Berlin, Germany) and by the State of Bavaria. The work leading to this publication also received support from the Innovative Medicines Initiative Joint Undertaking under grant agreement no. 115317 (DIRECT), the resources of which comprise financial contribution from the European Union's Seventh Framework Programme (FP7/2007-2013) and European Federation of Pharmaceutical Industries and Associations companies' in-kind contribution.

Duality of interest statement The authors declare that there is no duality of interest associated with this manuscript.

Contribution statement All authors made substantial contributions to one or more of the following: the study conception and design (BK, BT, $\mathrm{GG}, \mathrm{CHe}$ ); acquisition of data or analysis (BK, BT, WK, THG, CM, CHu, $\mathrm{WR}, \mathrm{AP}, \mathrm{CHe}$ ); and interpretation of data (BK, BT, PWF, MR, WR, $\mathrm{CHe}$ ). $\mathrm{CHe}$ drafted the article and all other authors contributed to revising the article critically for important intellectual content. All authors provided their final approval of the version to be published. BK is the guarantor of this work and responsible for the integrity of the work.

\section{References}

1. Donath MY, Shoelson SE (2011) Type 2 diabetes as an inflammatory disease. Nat Rev Immunol 11:98-107

2. Herder C, Carstensen M, Ouwens DM (2013) Anti-inflammatory cytokines and risk of type 2 diabetes. Diabetes Obes Metab 15(Suppl 3):39-50

3. Wang X, Bao W, Liu J et al (2013) Inflammatory markers and risk of type 2 diabetes: a systematic review and meta-analysis. Diabetes Care 36:166-175 
4. Larsen CM, Faulenbach M, Vaag A et al (2007) Interleukin-1receptor antagonist in type 2 diabetes mellitus. N Engl J Med 356:1517-1526

5. Goldfine AB, Fonseca V, Jablonski KA et al (2010) The effects of salsalate on glycaemic control in patients with type 2 diabetes: a randomized trial. Ann Intern Med 152:346-357

6. Cavelti-Weder C, Babians-Brunner A, Keller C et al (2012) Effects of gevokizumab on glycemia and inflammatory markers in type 2 diabetes. Diabetes Care 35:1654-1662

7. Ridker PM, Howard CP, Walter V et al (2012) Effects of interleukin- $1 \beta$ inhibition with canakinumab on hemoglobin A1c, lipids, C-reactive protein, interleukin-6, and fibrinogen: a phase IIb randomized, placebo-controlled trial. Circulation 126:2739-2748

8. Donath MY (2014) Targeting inflammation in the treatment of type 2 diabetes: time to start. Nat Rev Drug Discov 13:465-476

9. Tabák AG, Herder C, Rathmann W, Brunner EJ, Kivimäki M (2012) Prediabetes: a high-risk state for diabetes development. Lancet 379:2279-2290

10. International Diabetes Federation (2013) IDF diabetes atlas, 6th edn. International Diabetes Federation, Brussels

11. Xu Y, Wang L, He J et al (2013) Prevalence and control of diabetes in Chinese adults. JAMA 310:948-959

12. Selvin E, Parrinello CM, Sacks DB, Coresh J (2014) Trends in prevalence and control of diabetes in the United States, 19881994 and 1999-2010. Ann Intern Med 160:517-525

13. Müller S, Martin S, Koenig W et al (2002) Impaired glucose tolerance is associated with increased serum concentrations of interleukin 6 and co-regulated acute-phase proteins but not TNF-alpha or its receptors. Diabetologia 45:805-812

14. Herder C, Haastert B, Müller-Scholze S et al (2005) Association of systemic chemokine concentrations with impaired glucose tolerance and type 2 diabetes: results from the Cooperative Health Research in the Region of Augsburg Survey S4 (KORA S4). Diabetes 54(Suppl 2):S11-S17

15. Daimon M, Oizumi T, Saitoh T et al (2003) Decreased serum levels of adiponectin are a risk factor for the progression to type 2 diabetes in the Japanese population: the Funagata study. Diabetes Care 26: 2015-2020

16. Donahue RP, Stranges S, Rejman K, Rafalson LB, Dmochowski J, Trevisan M (2007) Elevated cystatin C concentration and progression to pre-diabetes: the Western New York study. Diabetes Care 30:1724-1729

17. Park K, Steffes M, Lee DH, Himes JH, Jacobs DR Jr (2009) Association of inflammation with worsening HOMA-insulin resistance. Diabetologia 52:2337-2344

18. Fall T, Hägg S, Mägi R et al (2013) The role of adiposity in cardiometabolic traits: a Mendelian randomization analysis. PLoS Med 10: e1001474

19. Rathmann W, Haastert B, Icks A et al (2003) High prevalence of undiagnosed diabetes mellitus in Southern Germany: target populations for efficient screening. The KORA survey 2000. Diabetologia 46:182-189

20. Rathmann W, Strassburger K, Heier M et al (2009) Incidence of type 2 diabetes in the elderly German population and the effect of clinical and lifestyle risk factors. KORA S4/F4 cohort study. Diabet Med 26:1212-1219

21. WHO (1999) Report of a WHO consultation: definition, diagnosis and classification of diabetes mellitus and its complications. World Health Organization, Geneva

22. John WG, Braconnier F, Miedema K, Aulesa C, Piras G (1997) Evaluation of the Menarini-Arkray HA 8140 hemoglobin A1c analyzer. Clin Chem 43:968-975
23. Thoelen AI, Moens M, Moerman J (2004) HbA1c: will the HbA1c auto-analyser HA-8160 (Menarini Diagnostics) imply a substantial improvement compared to the HA-8140? http://w1.uzleuven.be/ labo/Leermodule/EBLM_CAT/doc/CAT_040714_HbA1c.pdf, accessed 8 Jan 2015

24. Herder C, Hauner H, Haastert B et al (2006) Hypoadiponectinemia and proinflammatory state: two sides of the same coin?: results from the Cooperative Health Research in the Region of Augsburg Survey 4 (KORA S4). Diabetes Care 29:1626-1631

25. Gkrania-Klotsas E, Ye Z, Cooper AJ et al (2010) Differential white blood cell count and type 2 diabetes: systematic review and metaanalysis of cross-sectional and prospective studies. PLoS One 5: e13405

26. Lorenzo C, Hanley AJ, Haffner SM (2014) Differential white cell count and incident type 2 diabetes: the Insulin Resistance Atherosclerosis Study. Diabetologia 57:83-92

27. Tanigaki K, Vongpatanasin W, Barrera JA et al (2013) C-reactive protein causes insulin resistance in mice through Fcgamma receptor IIB-mediated inhibition of skeletal muscle glucose delivery. Diabetes 62:721-731

28. Brunner EJ, Kivimäki M, Witte DR et al (2008) Inflammation, insulin resistance, and diabetes - Mendelian randomization using CRP haplotypes points upstream. PLoS Med 5: e155

29. Lane T, Wassef N, Poole S et al (2014) Infusion of pharmaceuticalgrade natural human $\mathrm{C}$-reactive protein is not proinflammatory in healthy adult human volunteers. Circ Res 114:672-676

30. Marzi C, Huth C, Herder C et al (2013) Acute-phase serum amyloid A protein and its implication in the development of type 2 diabetes in the KORA S4/F4 study. Diabetes Care 36:1321-1326

31. Yang RZ, Lee MJ, Hu H et al (2006) Acute-phase serum amyloid A: an inflammatory adipokine and potential link between obesity and its metabolic complications. PLoS Med 3: e287

32. Filippin-Monteiro FB, de Oliveira EM, Sandri S, Knebel FH, Albuquerque RC, Campa A (2012) Serum amyloid A is a growth factor for 3T3-L1 adipocytes, inhibits differentiation and promotes insulin resistance. Int J Obes 36:1032-1039

33. Masters SL, Latz E, O'Neill LA (2011) The inflammasome in atherosclerosis and type 2 diabetes. Sci Transl Med 3:81ps17

34. Lee HM, Kim JJ, Kim HJ, Shong M, Ku BJ, Jo EK (2013) Upregulated NLRP3 inflammasome activation in patients with type 2 diabetes. Diabetes 62:194-204

35. Thorand B, Baumert J, Döring A et al (2006) Sex differences in the relation of body composition to markers of inflammation. Atherosclerosis 184:216-224

36. Holz T, Thorand B, Döring A, Schneider A, Meisinger C, Koenig W (2010) Markers of inflammation and weight change in middleaged adults: results from the prospective MONICA/KORA S3/F3 study. Obesity (Silver Spring) 18:2347-2353

37. InterAct Consortium, Langenberg C, Sharp SJ et al (2012) Longterm risk of incident type 2 diabetes and measures of overall and regional obesity: the EPIC-InterAct case-cohort study. PLoS Med 9: e1001230

38. Fizelova M, Stancakova A, Lorenzo C et al (2015) Glycated hemoglobin levels are mostly dependent on non-glycemic parameters in 9398 Finnish men without diabetes. J Clin Endocrinol Metab 100: 1989-1996

39. Herder C, Kowall B, Tabák AG, Rathmann W (2014) The potential of novel biomarkers to improve risk prediction of type 2 diabetes. Diabetologia 57:16-29

40. Koivula RW, Heggie A, Barnett A et al (2014) Discovery of biomarkers for glycaemic deterioration before and after the onset of type 2 diabetes: rationale and design of the epidemiological studies within the IMI DIRECT Consortium. Diabetologia 57:1132-1142 